\title{
Loss of Periodontal Attachment in HIV-Seropositive Military Personnel
}

\author{
Scott L. Tomar, ${ }^{*}$ Philip A. Swango,${ }^{\dagger}$ Dushanka V. Kleinman,${ }^{\dagger}$ and Brian A. Burt ${ }^{\star}$
}

THE CROSS-SECTIONAL RELATIONSHIP between severe loss of periodontal attachment (LPA) and worsening immune status due to HIV infection was evaluated in 474 HIVinfected subjects ( 416 men, 58 women) aged 18 to 49 years who had been classified at stages 1 through 6 of the Walter Reed Army Institute of Research (WR) Staging Classification System. LPA was measured at four sites per tooth using a manual probe; severe LPA was defined as $\geq 1$ site/subject exhibiting $\geq 5 \mathrm{~mm}$ LPA. Severe LPA was found in $94(20 \%)$ of the subjects. Modeling with multiple logistic regression analysis revealed that WR stage and peripheral CD4+ lymphocyte cell counts were not significant independent predictors of severe LPA. Severe LPA was more common in subjects at WR stage 5 or 6 who exhibited oral candidiasis (OC), a marker of immune system damage, than in persons at those WR stages without OC (odds ratio $=7.85 ; 95 \%$ confidence interval $(\mathrm{CI})=1.94-31.81)$. After the analysis controlled for WR stage, younger subjects receiving AZT had greater odds of severe LPA than sameage subjects not taking the drug (e.g., odds ratio for subjects aged 30 years $=2.59$; $95 \% \mathrm{CI}=1.22,5.49$ ). Other significant predictors in the model included male sex; retired military status; cigarette smoking; and presence of cratered, ulcerated, or necrotic interdental papillae. HIV-associated immune deficiency may be associated with localized severe LPA, but this may be an indirect association due to medication use, opportunistic infection, or other factors not captured by the WR staging system or peripheral CD4+ cell counts. Comparison with the estimated prevalence of HIV-associated periodontitis (HIV-P) in this population suggests that infected persons may experience severe LPA that does not necessarily have the clinical presentation of HIVP. J Periodontol 1995;66:421-428.

Key Words: Periodontal attachment; HIV infection/complications; immune system deficiency.

A rapidly progressive form of destructive periodontitis among patients infected with human immunodeficiency virus (HIV) was first observed at the University of California, San Francisco, in the mid-1980s..$^{1,2}$ This condition has been termed HIV-associated periodontitis, or HIV-P. ${ }^{3}$ Although HIV-P may be painful and may occasionally result in rapid tooth loss, ${ }^{3}$ its prevalence has been low in many HIV-seropositive study populations. ${ }^{4-6}$ Masouredis et al. ${ }^{5}$ have proposed operational case definitions for HIV$\mathrm{P}$ that may be applied either with or without periodontal probing. However, HIV-P may represent only one portion

*Currently, Epidemic Intelligence Service, Centers for Disease Control and Prevention, Atlanta, GA; previously, The University of Michigan School of Public Health, Ann Arbor, MI.

${ }^{+}$National Institute of Dental Research, National Institutes of Health, Bethesda, MD.

${ }^{*}$ The University of Michigan School of Public Health, Program in Dental Public Health, Ann Arbor, MI. of the clinical spectrum of periodontal disease in HIVinfected populations; more subtle destruction of periodontal tissues may go undetected or be considered "normal" adult periodontitis. Case definitions that address only the more unusual clinical periodontal manifestations may considerably underestimate the impact of HIV-associated immune deficiency on the periodontal health of infected populations. Few published studies have used objectively measurable clinical indicators to try to quantify the incidence or prevalence of periodontal destruction in HIV-infected populations. ${ }^{4,7}$

This study had the following objectives: 1) estimate the prevalence of severe LPA in an HIV-infected population; 2) compare the prevalence of severe LPA at various stages of HIV disease; 3 ) identify behavioral, clinical, and biological markers associated with increased odds of severe LPA; 4) construct statistical models to help explain the occurrence of severe LPA in HIV-infected individuals; 
Table 1. The Walter Reed Staging Classification for HIV Infection*

\begin{tabular}{|c|c|c|c|c|c|c|}
\hline Stage & $\mathrm{abHIV}^{\dagger}$ & $\begin{array}{c}\text { Chronic } \\
\text { Lymphadenopathy }\end{array}$ & $\begin{array}{c}\text { T Helper } \\
\text { Cells } / \mathrm{mm}^{3 \S}\end{array}$ & $\begin{array}{c}\text { Delayed } \\
\text { Hypersensitivity }\end{array}$ & Thrush & $\begin{array}{l}\text { Opportunistic } \\
\text { Infection" }\end{array}$ \\
\hline WR 0 & - & - & $>400$ & NL" & - & - \\
\hline WR 1 & + & - & $>400$ & $\mathrm{NL}$ & - & - \\
\hline WR 2 & + & + & $>400$ & NL & - & - \\
\hline WR 3 & + & $+1-$ & $<400$ & NL & - & - \\
\hline WR 4 & + & $+1-$ & $<400$ & $\mathbf{P}$ & - & - \\
\hline WR 5 & + & $+1-$ & $<400$ & $\mathrm{C}$ or & + & - \\
\hline WR 6 & + & $+1-$ & $<400$ & $\mathrm{P} / \mathrm{C}$ & $+1-$ & + \\
\hline
\end{tabular}

*Originally published in and reprinted with the permission of The New England Journal of Medicine; Redfield RR, Wright DC, Tramount EC. The Walter Reed Staging Classification for HTLV-III/LAV infection; volume 314; pages 131-132; copyright 1986 Massachusetts Medical Society.

Defined by the presence of antibody to the human immunodeficiency virus (HIV), as determined by the Western Blot technique $(+=$ present, $-=$ absent).

*Defined as 2 or more extrainguinal sites with lymph nodes $\geq 1 \mathrm{~cm}$ in diameter persistent for $>3$ months ( $+=$ present, $-=$ absent).

sQuantitative depletion $\left(<400\right.$ cells $\left./ \mathrm{mm}^{3}\right)$ must be present for at least 3 months for WR 3 classification.

INL: normal DHS is defined as an intact cutaneous anergy, defined as an intact cutaneous response to only 1 of the 4 test antigens; C: complete cutaneous anergy to the 4 test antigens.

"Clinical oral candidiasis including $+\mathrm{KOH}$ prep.

"Pneumocystis carinii pneumonia, CHS or disseminated toxoplasmosis, chronic cryptosporidiosis, candida esophagitis, disseminated histoplasmosis, CNS or disseminated cryptococcosis, disseminated atypical mycobacterial disease, extrapulmonary tuberculosis, disseminated nocardiosis, disseminated $\mathrm{CMV}$, or chronic mucocutaneous herpes simplex.

and 5) compare the severe LPA attributable in the study population to HIV-related damage to the immune system to the estimated prevalence of HIV-P.

\section{METHODS}

\section{Clinical Data}

Since October 1985, all active-duty U.S. military personnel and applicants for recruitment have received mandatory blood testing for HIV antibody. Persons who tested seropositive received medical evaluations and treatment at regional medical care centers and were scheduled for 6-month follow-up. These persons were given the opportunity to participate in a medical research protocol, conducted by the Walter Reed Army Institute of Research (WR), on the natural history of HIV infection. Scientists at the National Institute of Dental Research (NIDR) collaborated with Army investigators. NIDR's Oral Health Research Activity began operating in 1989. Details of this initiative were provided in an earlier report. ${ }^{8}$ The present study was cross-sectional in design and used data collected at baseline oral examinations conducted between 1989 and 1993.

Self-reported data were collected on demographic characteristics, dental care utilization, oral symptoms, mouth dryness and taste acuity, oral hygiene practices, and the use of tobacco and alcohol. Study participants were classified for HIV disease progression according to the WR Staging Classification System, ${ }^{9}$ which assigns subjects to one of six progressive disease stages based on clinical and laboratory presentations (Table 1). Staging of infected persons ranges from stages WR 1 and 2, in which patients are essentially asymptomatic with CD4+ T-lymphocyte counts greater than $400 \mathrm{cells} / \mathrm{mm}^{3}$, to progressively more advanced stages in which $\mathrm{CD} 4+\mathrm{T}$-lymphocyte counts are below 400 cells $/ \mathrm{mm}^{3}$ and clinical signs of damage to the immune system appear.

Loss of periodontal attachment, measured by manual probe at four sites per tooth as the distance in millimeters from the cemento-enamel junction (CEJ) of a tooth to the bottom of the gingival sulcus or pocket, was determined for all teeth except third molars. LPA and gingival bleeding were measured at disto-buccal, mid-buccal, mesiobuccal, and mid-lingual sites. Plaque and calculus indices $^{10,11}$ were used to score the same four sites per tooth on six teeth per subject (the four first molars and the maxillary right and mandibular left central incisors). All available interdental papillae anterior to the second molars were examined for signs of inflammation and destruction, for a maximum of 13 papillary units per arch (buccal and lingual papillae were scored as one unit). Erythematous gingival banding was assessed for all teeth except third molars and all subjects were examined for lesions on oral soft tissue. Oral examinations were conducted through standardized examination protocol by a specialist in oral medicine trained in the diagnosis of HIV-related conditions and the use of oral epidemiological indices.

Currently, destructive periodontitis has no universally accepted operational definition, particularly for cross-sectional investigations. Severe LPA was defined in this study as the presence of one or more periodontal sites with LPA $\geq 5 \mathrm{~mm}$. Referents were subjects whose most severe LPA was $<5 \mathrm{~mm}$.

The primary predictors considered in this study were measures of immune system damage secondary to HIV infection: WR stage of HIV disease (see Table 1) and peripheral CD4+ lymphocyte counts taken at the time of the oral examination. Other predictors and potential confounders included age and other demographic variables, self-reported oral hygiene practices and utilization of den- 
tal care services, tobacco use, use of medications, clinical measures of oral hygiene, and signs of pathology on periodontal and oral soft tissue.

Because the association between age and cumulative LPA was strong and the sample size and age distribution precluded valid inferences on older persons, analysis was restricted to subjects $<50$ years of age.

\section{Statistical Analysis}

Statistical analysis for each outcome variable was performed by using both bivariate contingency table analysis and multiple logistic regression; estimated prevalence odds ratios were the primary measures of association. Crude bivariate analysis was performed for each predictor and outcome variable, followed by age- and WR-stagestratified analysis to assess interaction and control for confounding. Adjusted odds ratio estimates in the stratified analyses were calculated by using the method of Mantel and Haenszel. ${ }^{12}$ Guided by the results of bivariate analyses, multiple logistic regression models were constructed to allow assessment of the independent effect of the predictor variables by simultaneously controlling for confounders and other correlates. Potential effect modification was assessed through the inclusion of interaction terms. The statistical significance of main effect and interaction terms in the logistic regression models was assessed through the use of likelihood ratio tests. ${ }^{13}$ The goodness-of-fit of the models was evaluated through the use of the Hosmer-Lemeshow test procedure. ${ }^{13}$

The prevalence of HIV-P in the study population was estimated by applying the criteria of Masouredis et al..$^{5}$ to measured clinical indicators (including periodontal probing) (Table 2). Although this case definition may have been intended for application at the time of clinical examination, all applicable indicators had been individually measured on participants in the present study, thereby allowing a retrospective application of the case definition.

Among the cases of severe LPA observed in the study population, the proportion that was attributable to profound HIV-associated immune deficiency was assessed through the application of the following formula: ${ }^{14}$

$$
\mathrm{AP}_{\mathrm{T}}=\left[(\mathrm{RR}-1) \mathrm{P}_{1}\right] / \mathrm{RR}
$$

where $\mathrm{AP}_{\mathrm{T}}=$ the proportion of all cases of severe LPA in the population attributable to profound immunodeficiency, $R R=$ the incidence rate ratio (estimated by the prevalence odds ratio), and $P_{1}=$ the proportion of the cases that had profound immunodeficiency.

The proportion of severe LPA cases attributable to profound immune deficiency in this study population was estimated by using the age-adjusted odds ratios associated with peripheral CD4+ T-lymphocyte counts $<300$ cells/ $\mathrm{mm}^{3}$. (Although low CD4+ T-lymphocyte counts did not contribute significantly to the multivariate modeling of severe LPA, it was a significant correlate in bivariate anal-
Table 2. Operational Definitions for a Diagnosis of HIV-Associated Gingivitis (HIV-G) or HIV-Associated Periodontitis (HIV-P)*

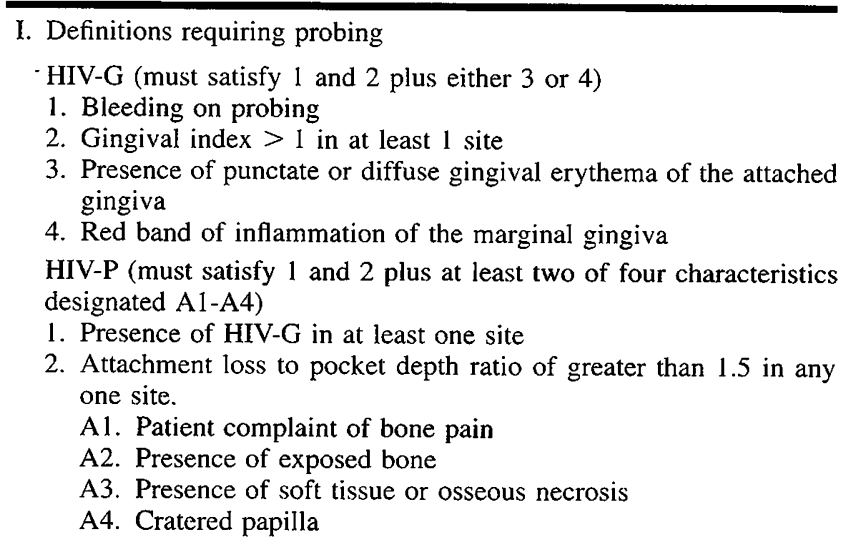

II. Definitions not requiring probing

HIV-G (must satisfy 1 plus either 2 or 3 )

1. Gingival index $>1$ in at least 1 site

2. Presence of punctate or diffuse gingival erythema of the attached gingiva

3. Red band of inflammation of the marginal gingiva

HIV-P (must satisfy 1 plus two of four characteristics designated AlA4)

1. Presence of HIV-G in at least one site A1. Patient complaint of bone pain

A2. Presence of exposed bone

A3. Presence of soft tissue or osseous necrosis A4. Cratered papilla

* Originally published in and reprinted with the permission of Journal of the Acquired Immune Deficiency Syndrome; Masouredis CM, Katz MH, Greenspan D, et al. Prevalence of HIV-associated periodontitis and gingivitis in HIV-infected patients attending an AIDS clinic; 1992;5: 479-483.

ysis and serves as a useful and valid marker for profound immune system damage. ${ }^{15}$ )

\section{RESULTS}

A total of 619 records were identified in the files of the Oral Research Activity as of January 1993. Of these, 56 (9.0\%) had no data on periodontal attachment loss and an additional 64 records $(10.3 \%)$ were missing data on HIV disease staging or other important predictors. Twenty-two (22) subjects $(3.6 \%)$ were excluded because they were over the age of 49 years, and $3 \mathrm{HIV}$-seronegative subjects were omitted from analysis.

Characteristics of the remaining 474 study subjects are presented in Table 3. Subjects missing periodontal data tended to be older than subjects with complete periodontal information (data not shown). Subjects not included in analysis were more likely than included subjects to be retired from military service rather than in active duty and were more likely to have been at more advanced stages of HIV disease. The two groups were similar on most other demographic characteristics.

Overall, 94 (19.8\%) study subjects had severe LPA. Men were more likely than women to have severe LPA, and subjects retired from military service were more like- 
Table 3. Selected Characteristics of the Study Subjects $(N=474)$

\begin{tabular}{|c|c|c|}
\hline & Number & $\%$ \\
\hline \multicolumn{3}{|l|}{ Age (years) } \\
\hline $18-29$ & 193 & 40.7 \\
\hline $30-39$ & 221 & 46.6 \\
\hline $40-49$ & 60 & 12.7 \\
\hline \multicolumn{3}{|l|}{ Education (years) } \\
\hline$\leq 12$ & 199 & 42.0 \\
\hline $13-16$ & 235 & 49.6 \\
\hline$>17$ & 40 & 8.4 \\
\hline \multicolumn{3}{|l|}{ Sex } \\
\hline Female & 58 & .12 .2 \\
\hline Male & 416 & 87.8 \\
\hline \multicolumn{3}{|l|}{ Race } \\
\hline Black & 249 & 52.5 \\
\hline White & 187 & 39.5 \\
\hline Other/unknown & 38 & 8.0 \\
\hline \multicolumn{3}{|l|}{ Country of birth* } \\
\hline USA & 430 & 91.3 \\
\hline Other & 41 & 8.7 \\
\hline \multicolumn{3}{|l|}{ Military status } \\
\hline Active duty & 244 & 51.5 \\
\hline Retired & 183 & 38.6 \\
\hline Dependent/other & 47 & 9.9 \\
\hline \multicolumn{3}{|l|}{ Walter Reed Stage } \\
\hline 1 & 75 & 15.8 \\
\hline 2 & 166 & 35.0 \\
\hline 3 & 136 & 28.7 \\
\hline 4 & 30 & 6.3 \\
\hline 5 & 46 & 9.7 \\
\hline 6 & 21 & 4.4 \\
\hline
\end{tabular}

*Data on country of birth are missing for 3 subjects.

ly to have experienced severe LPA than active duty personnel and dependents (Table 4). The odds of severe LPA tended to increase with worsening severity of HIV disease. When the analysis controlled for age or WR stage, subjects who first tested seropositive for HIV in 1988 or later were significantly less likely to have severe LPA, and duration of HIV infection was not significantly associated with severe LPA. Both current and former cigarette smokers were significantly more likely to have severe LPA than nonsmokers. In all age groups, subjects with plaque or calculus index scores above the group median were more likely to have severe LPA. Gingival bleeding and severe LPA showed an effect modification of age; subjects $\geq 30$ years of age who had higher mean gingival bleeding index scores were less likely to have severe LPA than same-aged subjects with lower bleeding scores, although the stratum-specific odds ratio estimates did not differ significantly from unity.

Severe LPA was significantly associated with the presence of a high number of erythematous interdental papillae or one or more edematous or cratered papillae. Red gingival banding showed no association with severe LPA. The presence of one or more ulcerated or necrotic interdental papillae was strongly associated with severe LPA, but only seven subjects exhibited these conditions.
In every age group, subjects with oral candidiasis (OC), defined as the presence of erythematous or pseudomembranous intraoral candidal infection, were more likely to have severe LPA than subjects without OC. The strength of association between OC and severe LPA increased with advancing stage of HIV disease (Fig. 1). The odds ratio estimates for stages WR 1 through 4 were not statistically different from unity, although the Breslow-Day test for homogeneity of the odds ratios $\left(\chi^{2}{ }_{\mathrm{BD}}=6.18, \mathrm{df}=2 ; P\right.$ $=0.046)$ indicated a probable effect modification of stage of HIV disease on the association between OC and severe LPA. Angular cheilitis tended to be associated with increased odds of severe LPA, although this condition was observed in only 17 subjects. Subjects at stages WR 3 or higher who had intraoral hairy leukoplakia, a lesion strongly associated with immunosuppression and predictive of AIDS, ${ }^{16}$ were more likely to exhibit severe LPA than same-staged subjects without this lesion.

Subjects under 40 years of age who were taking AZT/ zidovudine were more likely to have severe LPA, although this relation diminished with increasing age. Subjects at stages WR 1 or WR 2 who were taking AZT/ zidovudine were twice as likely to have severe LPA as subjects at these stages not taking these medications, although the confidence intervals for the odds ratio estimates included 1.0 in this stratified analysis. Small numbers of subjects in each cell prohibited a simultaneous stratification on both age and WR stage for these variables.

In multiple logistic regression modeling, the strongest correlate of severe LPA was age, which was controlled for by its inclusion in multivariate models (Table 5). After the analysis controlled for age, WR stage of disease, and other variables in the model, men were about three times as likely as women to have severe LPA, and retired subjects were about twice as likely as active-duty subjects to have severe LPA. WR stage of HIV disease was not a significant independent correlate of severe LPA, but WR stage of disease and the presence of oral candidiasis strongly affected the likelihood of severe LPA. Multiple logistic modeling indicated that subjects at WR stages 5 and 6 who had OC were over seven times more likely to have severe LPA than subjects at those stages without OC.

Nine subjects (1.9\%) were classified as having HIV-P, according to the case definition of Masouredis et al. ${ }^{5}$ An estimated $16.6 \%$ (16 persons) of the severe LPA seen in this study population was attributable to profound HIVassociated immune deficiency (peripheral CD4 + T-lymphocyte counts $<300$ cells $/ \mathrm{mm}^{3}$ ) (Table 6).

\section{DISCUSSION}

Severe loss of periodontal attachment was not directly associated with HIV-related immunosuppression after the analysis controlled for other significantly associated vari- 
Table 4. Odds Ratio Estimates for Loss of Periodontal Attachment (LPA) $\geq 5 \mathrm{~mm}$ Associated With Selected Variables, Bivariate Analysis

\begin{tabular}{|c|c|c|c|c|}
\hline Variable & $\mathrm{N}$ & $\begin{array}{c}\text { Prevalence of } \\
\text { Severe } \\
\text { LPA (\%) }\end{array}$ & Odds Ratio* & 95\% C.I. \\
\hline Total & 474 & 19.8 & & \\
\hline \multicolumn{5}{|l|}{ Sex } \\
\hline Female & 58 & 10.3 & 1.00 & \\
\hline Male & 416 & 21.2 & 2.66 & $1.13,6.25$ \\
\hline \multicolumn{5}{|l|}{ Military status } \\
\hline Active duty & 244 & 14.8 & 1.00 & \\
\hline Retired & 183 & 27.9 & 2.09 & $1.24, \quad 3.56$ \\
\hline Dependent/other & 47 & 14.9 & 0.90 & $0.32, \quad 2.39$ \\
\hline \multicolumn{5}{|l|}{ Walter Reed Stage } \\
\hline $1-2$ & 241 & 19.3 & 1.00 & \\
\hline $3-4$ & 166 & 21.7 & 1.26 & $0.73, \quad 2.19$ \\
\hline $5-6$ & 67 & 28.4 & 1.80 & $0.91, \quad 3.66$ \\
\hline \multicolumn{5}{|c|}{ CD4+ T-lymphocytes $/ \mathrm{mm}^{3}$} \\
\hline$\geq 500$ & 159 & 14.5 & 1.00 & \\
\hline $300-499$ & 156 & 18.6 & 1.24 & $0.64, \quad 2.39$ \\
\hline$\leq 299$ & 159 & 26.4 & 2.12 & $1.00, \quad 3.47$ \\
\hline \multicolumn{5}{|c|}{ Date of presumed seroconversion } \\
\hline Before 1988 & 248 & 24.2 & 1.00 & \\
\hline 1988 and later & 226 & 15.0 & 0.61 & $0.38, \quad 0.97$ \\
\hline \multicolumn{5}{|l|}{ Cigarette smoking } \\
\hline Never smoked & 181 & 11.6 & 1.00 & \\
\hline Former smoker & 90 & 24.4 & 2.37 & $1.15,4.90$ \\
\hline Current smoker & 203 & 25.1 & 2.63 & $1.45, \quad 4.79$ \\
\hline \multicolumn{5}{|c|}{ Mean plaque index score } \\
\hline$\leq$ Median & 260 & 15.0 & 1.00 & \\
\hline Above median & 214 & 25.7 & 2.12 & $1.33, \quad 3.38$ \\
\hline \multicolumn{5}{|c|}{ Mean calculus index score } \\
\hline$\leq$ Median & 265 & 14.7 & 1.00 & \\
\hline Above median & 209 & 26.3 & 2.17 & $1.36, \quad 3.46$ \\
\hline \multicolumn{5}{|c|}{ Mean gingival bleeding index score } \\
\hline$\leq$ Median & 249 & 22.5 & 1.00 & \\
\hline Above median & 225 & 16.9 & 0.77 & $0.48, \quad 1.23$ \\
\hline \multicolumn{5}{|c|}{ Erythematous interdental papillae } \\
\hline$<15$ & 359 & 14.5 & 1.00 & \\
\hline$\geq 15$ & 115 & 36.5 & 3.61 & $2.25, \quad 5.81$ \\
\hline \multicolumn{5}{|c|}{ Edematous interdental papillae } \\
\hline 0 & 313 & 13.4 & 1.00 & \\
\hline$\geq 1$ & 161 & 32.3 & 3.06 & $1.93, \quad 4.85$ \\
\hline \multicolumn{5}{|c|}{ Cratered interdental papillae } \\
\hline 0 & 394 & 12.2 & 1.00 & \\
\hline$\geq 1$ & 80 & 57.5 & 9.89 & $6.03,16.23$ \\
\hline
\end{tabular}

*Adjusted for age.

ables. However, OC, which showed a considerable interaction with WR staging on the odds of severe LPA, was itself strongly associated with advanced stages of HIV.

Given the cross-sectional nature of this study, few firm conclusions may be reached. However, the data do suggest that Candida species may be involved in the pathogenesis of periodontal destruction in HIV-infected subjects. This suggestion would be consistent with the findings of Zambon and colleagues, ${ }^{6}$ who reported higher carriage rates of subgingival yeasts in subgingival plaques in AIDS patients than in patients without AIDS. Alternatively, $\mathrm{OC}$ infection may be a marker for immune disorders not measured in this study; mucocutaneous candidiasis in the presence of depressed CD4+ T-lymphocyte counts has been considered a direct measure of immune system damage. ${ }^{17}$ This interpretation would be consistent with the relation between hairy leukoplakia, which is associated with immune deficiency, ${ }^{16}$ and severe LPA.

The significant association between AZT/zidovudine use and severe LPA among younger subjects may indicate 


\section{Hairy Leukoplakia (HL)}

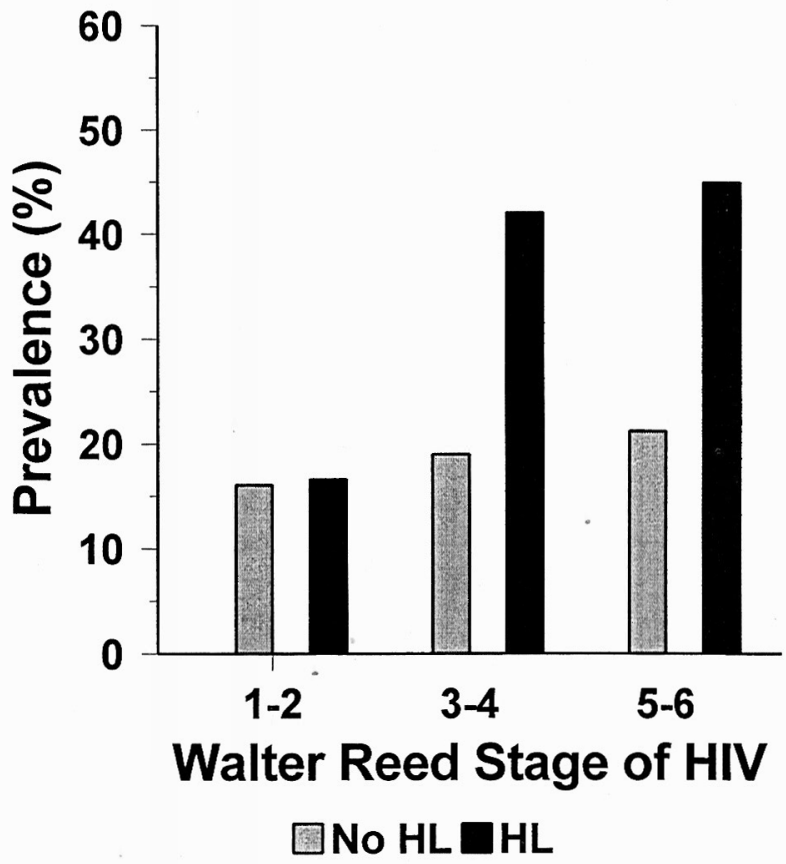

\section{Oral Candidiasis (OC)}

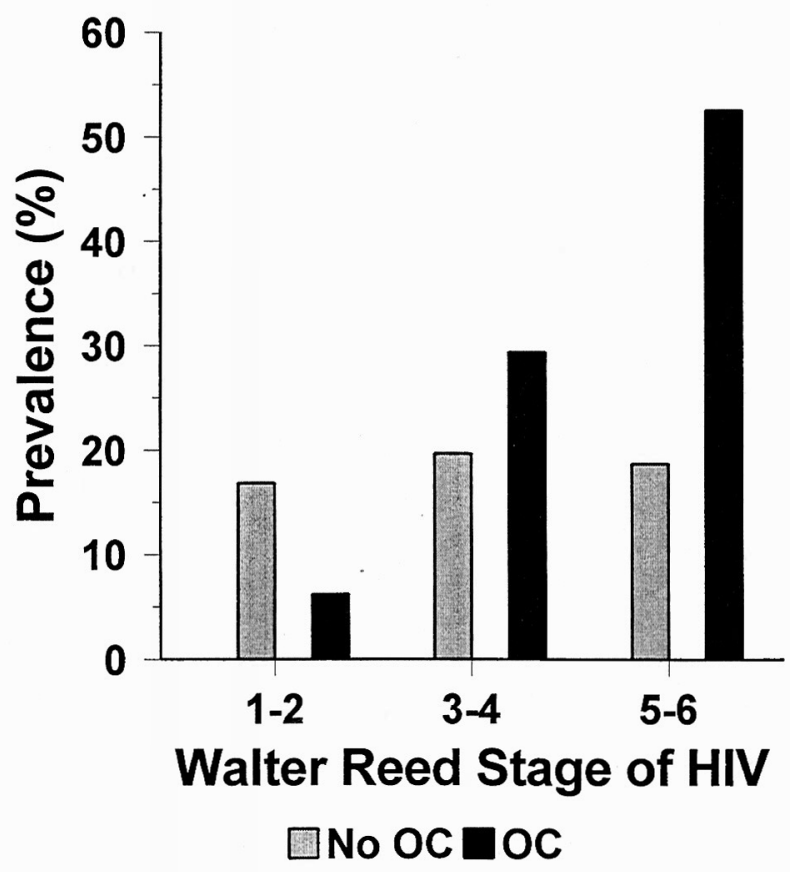

Figure 1. Prevalence of severe LPA ( $\geq 5 \mathrm{~mm})$, by Walter Reed Stage of HIV disease and presence of hairy leukoplakia or oral candidiasis.

the strong association between its use and the severity of HIV disease. Yet AZT use was more strongly associated with severe LPA than either of the two primary markers of immunosuppression in this study. This finding may indicate a possible pathogenic role for AZT in periodontal destruction, perhaps through interference of neutrophil
Table 5. Odds Ratio Estimates for LPA $\geq 5 \mathrm{~mm}$ Associated With Selected Variables, Multiple Logistic Regression Modeling

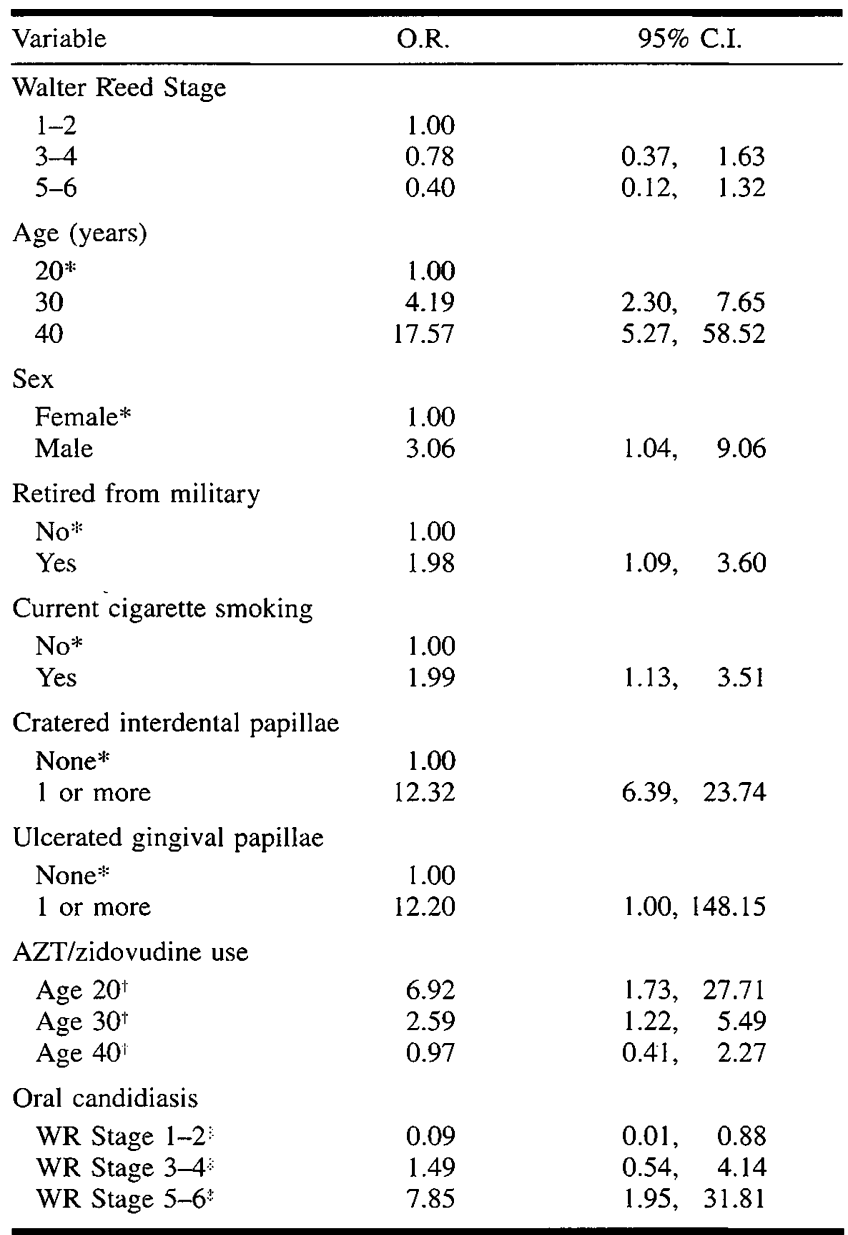

*Reference group.

${ }^{\dagger}$ Odds ratio estimates in reference to same-aged subjects not taking AZT/ Zidovudine.

Odds ratio estimates in reference to same-staged subjects without oral candidiasis.

Table 6. Association Between Peripheral CD4+ T-Lymphocyte Counts and LPA $\geq 5 \mathrm{~mm}$ and Proportion of Cases of LPA $\geq 5 \mathrm{~mm}$ Attributable to Peripheral CD4 + T-Lymphocyte Count $<300$ Cells/ $\mathbf{m m}^{\mathbf{3}}$

\begin{tabular}{|c|c|c|c|c|}
\hline \multirow{3}{*}{$\mathrm{LPA} \geq 5 \mathrm{~mm}$} & \multicolumn{4}{|c|}{$\mathrm{CD} 4+<300$ cells $/ \mathrm{mm}^{3}$} \\
\hline & & Yes & No & \multirow[b]{2}{*}{94} \\
\hline & \multirow{3}{*}{$\begin{array}{l}\text { Yes } \\
\text { No }\end{array}$} & 42 & 52 & \\
\hline & & 117 & 263 & 380 \\
\hline & & 159 & 315 & 474 \\
\hline
\end{tabular}

Crude odds ratio $(\mathrm{OR})=1.82$.

Age-adjusted odds ratio $\left(\mathrm{OR}_{\mathrm{udj}}\right)=1.59$.

Proportion of cases with $\mathrm{CD} 4+<300$ cells $/ \mathrm{mm}^{3}\left(\mathrm{P}_{1}\right)=42 / 94=0.45$.

$\mathrm{AP}_{\mathrm{T}}=\left[\left(\mathrm{OR}_{\mathrm{adj}}-1\right) \mathrm{P}_{\mathrm{j}}\right] / \mathrm{OR}_{\mathrm{adj}}$

$=[0.59 \times 0.45] / 1.59$

$=.166$.

Number of cases attributable to $\mathrm{CD} 4+<300$ cells $/ \mathrm{mm}^{3}=.166 \times 94$

$\approx 16$ persons. 
function and induced neutropenia. ${ }^{18,19}$ The neutrophil has been characterized as the most important cell in host defense against bacterial and certain fungal infections, and abnormal neutrophil function is the major host factor implicated in periodontitis. ${ }^{20}$ The study design and the absence of data on neutrophil numbers or function in this study do not permit a more detailed investigation of this hypothesis. The interaction between AZT use and age on the odds of severe LPA, with the strongest association between AZT and LPA seen among younger subjects, may indicate that the effects may be more noticeable at an age where moderate to severe LPA is usually quite rare, but may be difficult to separate from the lifetime accumulation of LPA seen in older individuals. The available data did not allow us to assess potential confounding of the association between AZT use and severe LPA due to medical indicators of immune dysfunction; i.e., physicians may have prescribed AZT for conditions other than depletion of CD4+ T-lymphocytes. AZT use was also based on self-report by study participants and may have been subject to misclassification, particularly if some subjects were participants in blinded clinical protocols.

After other factors were controlled for, both current and former smokers were more likely to have severe LPA than those who had never smoked. This finding was consistent with other epidemiological studies, ${ }^{21,22}$ although the pathogenic mechanism has not yet been elaborated.

Retirement status, which was associated with nearly twice the odds of severe LPA, may reflect a possible selfselection bias. "Retired" classification in this population does not necessarily mean retirement after 20 years of active duty; some subjects were medically retired as a result of HIV-related disability, even though they had less than 20 years of service. These subjects may have participated in the study secondary to HIV-associated oral conditions, including periodontal pathology. The finding may also indicate that WR staging is not particularly sensitive in describing the degree of morbidity, at least in terms of factors involved in periodontal destruction.

The strong association between severe LPA and the presence of interdental papillary cratering, ulceration or necrosis, and erythema may indicate that those who experience one form of gingival inflammation or destruction may be more likely to experience others as well. None of these interdental papillary clinical signs were associated with CD4+ T-lymphocyte count, WR staging, or AZT use.

Although men may tend to have slightly more severe LPA than women, ${ }^{23}$ it is difficult to explain the nearly three-fold increase in the odds of severe LPA for males in this study after the analysis controlled for age, HIV disease stage, and other variables in the multivariate models. When the analysis controlled for mean plaque index score (which did not contribute significantly in multivariate modeling) to assess whether the gender discrepancy could be due to differences in oral hygiene, the OR estimate was only slightly attenuated. Perhaps HIV-diseaserelated periodontal manifestations differ between the sexes, or the discrepancy may simply reflect the composition of this particular study population.

The findings of this study are generally consistent with other reports. In a 20-month longitudinal study of relative attachment loss (RAL) in HIV-seropositive and HIV-seronegative men, Barr and colleagues ${ }^{24}$ found that low CD4+ T-lymphocyte counts were associated with increased likelihood of RAL in subjects aged 35 years and older. Friedman et al. ${ }^{25}$ found that AIDS patients in a clinic for infectious disease had slightly higher mean attachment loss than HIV-seropositive patients who did not have AIDS, although small numbers of subjects resulted in relatively imprecise estimates and an absence of statistical significance.

The application of the case definition for HIV-P resulted in nine subjects being classified as "diseased." The study's relatively limited definition of profound immune system damage secondary to HIV infection indicated that about 16 of the 94 cases of severe LPA in this study population were attributable to advanced immune deficiency. This number is probably an underestimate, since it does not include other HIV-associated factors that were significantly associated with severe LPA (such as the presence of $\mathrm{OC}$ ), and since it uses odds ratio estimates based on an exposure classification that characterizes many of the HIV-infected individuals as "unexposed" (no true control group is used). Although the calculation of the attributable proportion used a simplistic definition of exposure, it illustrates that HIV-P may represent only a portion of the clinical spectrum of HIV-associated periodontal destruction. Case definitions that are restricted to the more severe periodontal manifestations may considerably underestimate the impact of HIV infection on the periodontal health of infected populations.

Bias may have been introduced by excluding subjects who had missing periodontal data. Although the study could not definitively explain the absence of periodontal data for 56 subjects, notes in a number of records indicated that the periodontal probings were not performed because of either extremely painful periodontal tissues, excessive bleeding that made probing measurements impossible, or systemic morbidity that resulted in a curtailed oral examination. In addition, subjects excluded from analysis were more likely to be retired from military service, and tended to be at more advanced stages of HIV disease than those included in analysis. The inclusion of these subjects would likely have strengthened the association between markers of HIV-associated immune system damage and severe LPA.

Although the cross-sectional design of this study precludes firm conclusions, the current investigation suggests that HIV-associated immune deficiency may be associated 
with severe LPA that does not necessarily present as the HIV-associated periodontitis described by other researchers. $^{2}$ Some of the "conventional periodontitis" observed in HIV-infected populations ${ }^{5}$ may be part of the clinical spectrum of HIV-associated periodontal disease. Neither the WR Staging Classification System nor peripheral CD4+ T-lymphocyte counts may be adequate markers for the immune system changes involved in the pathogenesis of periodontal destruction in HIV-infected persons.

\section{Acknowledgments}

This study was funded in part by grant T32 DE 07157 from the National Institute of Dental Research. Portions of this paper were presented in March 1994 at the annual meeting of the International Association for Dental Research, Seattle, Washington. We are grateful to Dr. Joseph Konzelman for his invaluable contribution to this project. Many thanks to Drs. Stephen Eklund, Michael A. Schork, and MaryFran Sowers for their considerable assistance and advice.

\section{REFERENCES}

1. Winkler JR, Murray PA, Greenspan D. AIDS-virus associated periodontal disease. J Dent Res 1986;65(Spec. Issue):741 (Abstr. 139).

2. Winkler JR, Murray PA. Periodontal disease: a potential intraoral expression of AIDS may be rapidly progressive periodontitis. Calif Dent Assoc J 1987;15:20-24.

3. Winkler JR, Grassi M, Murray PA. Clinical description and etiology of HIV-associated periodontal diseases. In: Robertson PB, Greenspan JS, eds. Perspectives on Oral Manifestations of AIDS: Diagnosis and Management of HIV-Associated Infections. Littleton, MA: PSG Publishing; 1988;49-70.

4. Drinkard CR, Decher L, Little JW, et al. Periodontal status of individuals in early stages of human immunodeficiency virus infection. Community Dent Oral Epidemiol 1991;19:281-285.

5. Masouredis CM, Katz MH, Greenspan D, et al. Prevalence of HIVassociated periodontitis and gingivitis in HIV-infected patients attending an AIDS clinic. J Acquir Immune Defic Syndr 1992;5:479483.

6. Zambon JJ, Reynolds HS, Genco RJ. Studies of the subgingival microflora in patients with acquired immunodeficiency syndrome. $J$ Periodontol 1990;61:699-704.

7. Cornick DER, Robinson PG. A three year study of the periodontal condition in HIV positive men. J Dent Res 1991;70(Spec. Issue): 688 (Abstr. 154).

8. Swango PA, Kleinman DV, Konzelman JL. HIV and periodontal health: a study of military personnel with HIV. $J$ Am Dent Assoc 1991;122:(8)49-54.
9. Redfield RR, Wright DC, Tramont EC. The Walter Reed staging classification for HTLV-III/LAV infection. New Engl J Med 1986; 314:131-132.

10. Löe H, Silness J. Periodontal disease in pregnancy. I. Prevalence and severity. Acta Odontol Scand 1963;21:533-551.

11. Löe $\mathbf{H}$. The gingival index, the plaque index, and the retention index systems. J Periodontol 1967;38(Suppl.):610-616.

12. Mantel N, Haenszel W. Statistical aspects of the analysis of data from retrospective studies of disease. J Natl Cancer Inst 1959;22: 719-748.

13. Hosmer DW, Lemeshow S. Applied Logistic Regression. New York: John Wiley \& Sons; 1989;25-36:135-175.

14. Rothman KJ. Modern Epidemiology. Boston: Little, Brown, and Co.; 1986;38-39.

15. Burke DS, Redfield RR. The stages of HIV infection: A predictable progressive disease. In: Rapoza $\mathrm{N}$, ed. HIV Infection and Disease. Chicago: American Medical Association; 1989;1-11.

16. Greenspan D, Greenspan JS, Hearst NG, et al. Relation of oral hairy leukoplakia to infection with the human immunodeficiency virus and the risk of developing AIDS. J Infect Dis 1987;155:475-481.

17. Gardner LI, Brundage JF, McNeil JG, et al. Predictors of HIV-1 disease progression in early- and late-stage patients: the US Army natural history cohort. J Acquir Immune Defic Syndr 1992;5:782793.

18. Ellis M, Gupta S, Galant S, et al. Impaired neutrophil function in patients with AIDS or AIDS-related complex: a comprehensive evaluation. J Infect Dis 1988;158:1268-1276.

19. Israel DS, Plaisance KI. Neutropenia in patients infected with human immunodeficiency virus. Clin Pharm 1991;10:268-279.

20. Van Dyke TE, Hoop GA. Neutrophil function and oral disease. Crit Rev Oral Biol Med 1990;1:117-133.

21. Ismail AI, Burt BA, Eklund SA. Epidemiologic patterns of smoking and periodontal disease in the United States. J Am Dent Assoc 1983; 106:617-621.

22. Bergstrom J. Cigarette smoking as risk factor in chronic periodontal disease. Community Dent Oral Epidemiol 1989;17:245-247.

23. National Institute of Dental Research. Oral Health of United States Adults. The National Survey of Oral Health in US Employed Adults and Seniors: 1985-1986. Washington, DC: National Institutes of Health, 1987; NIH pub. no. 87-2868.

24. Barr C, Lopez MR, Rua-Dobles A. Periodontal changes by HIV serostatus in a cohort of homosexual and bisexual men. $J$ Clin Periodontol 1992;19:794-801.

25. Friedman RB, Gunsolley J, Gentry A, Dinius A, Kaplowitz L, Settle J. Periodontal status of HIV-seropositive and AIDS patients. J Periodontol 1991;62:623-627.

Send reprint requests to: Dr. Scott L. Tomar, Office on Smoking and Health, National Center for Chronic Disease Prevention and Health Promotion, Centers for Disease Control and Prevention, 4770 Buford Highway N.E., Mail Stop K-50, Atlanta, GA 30341-3724.

Accepted for publication October 17, 1994. 